\title{
Two Error Models for Calibrating SCARA Robots based on the MDH Model
}

\author{
Xiaolong $\mathrm{Li}^{1}$, Hong $\mathrm{Hu}^{1}$ and Wenhua Ding ${ }^{2}$ \\ ${ }^{1}$ Harbin Institute of Technology Shenzhen Graduate School, China \\ ${ }^{2}$ Shenzhen Colibri Technologies Co. Ltd., China
}

\begin{abstract}
This paper describes the process of using two error models for calibrating Selective Compliance Assembly Robot Arm (SCARA) robots based on the modified Denavit-Hartenberg(MDH) model, with the aim of improving the robot's accuracy. One of the error models is the position error model, which uses robot position errors with respect to an accurate robot base frame built before the measurement commenced. The other model is the distance error model, which uses only the robot moving distance to calculate errors. Because calibration requires the end-effector to be accurately measured, a laser tracker was used to measure the robot position and distance errors. After calibrating the robot and, the end-effector locations were measured again compensating the error models' parameters obtained from the calibration. The finding is that the robot's accuracy improved greatly after compensating the calibrated parameters.
\end{abstract}

\section{Introduction}

Industrial robots usually work from one taught point to another taught point, which makes them highly repeatable although their accuracy may be poor due mainly to geometric errors.

The good news is that the accuracy can be improved by calibrating the robots. The two most important parts of robot calibration are building an accurate model and a valid method to measure the robot's position. Although some researchers, such as Marko Švaco[1], have calibrated robots using the Denavit-Hartenberg (DH) model, it is not good enough for some robots that have parallel links. So Hayati[2]proposed a model called the MDH model, which many researchers now use to calibrate robots. Some researchers have proposed methods for third level calibrations, such as Albert Nubiola[3, 4] who introduced the temperature influence. Wang Zhen Hua [5] proposed a method to calibrate robots without building a base frame by using the distance errors of moving robots. Zhou Jian[6] proposed a pose selection algorithm that allows one to select a given number of optimal poses out of a large set of previously measured poses to avoid local convergence.

Some researchers concentrated on the measure methods, such as: laser tracker[7], coordinate measuring machine (CMM)[8, 9], telescoping ballbar[4], and the vision system[10]. There are also some researchers who use novel methods such as a probe and a ball[11] to measure the robot's moving distance, and XiNing[12] used a laser and a PSD to calibrate robots.

In this paper, two error models are used to calibrate a
SCARA robot. One is a position error model and the other is a distance error model, both of which are based on the MDH model.

The paper is organized into the following sections: section 2 proposes a kinematic model of the industrial robot called $\mathrm{MDH}$ model; section 3 presents the two calibration error models; section 4 presents the experiments and results; and section 5 is the conclusion.

\section{$2 \mathrm{MDH}$ Model}

A robot model must be built before the robot can be calibrated. The DH model is the most popular model as its four parameters can express two link positions very well. But the DH model will results in singularities with respect to two adjacent parallel links, even though a very small parallel error will cause a large deviation. So a $\mathrm{MDH}$ model developed by Hayati[2] was used, involving a new parameter termed $\beta$ as the angle link $i$ rotates around the $y$ axis, written as $\operatorname{Rot}(y, \beta)$. The $\mathrm{MDH}$ model can be expressed as follows:

$$
\left[\begin{array}{cccc}
{ }_{i}^{i-1} A={ }_{i}^{i-1} T \cdot R_{Y}(\beta)= \\
\cos \theta \cos \beta & -\sin \theta & \sin \beta \cos \theta & a \\
\sin \alpha \sin \beta+\cos \alpha \cos \beta \sin \theta & \cos \alpha \cos \theta & \cos \alpha \sin \beta \sin \theta-\cos \beta \sin \alpha & -d \sin \alpha \\
\cos \beta \sin \alpha \sin \theta-\cos \alpha \sin \theta & \sin \alpha \cos \theta & \cos \alpha \cos \beta+\sin \alpha \sin \beta \sin \theta & d \cos \alpha \\
0 & 0 & 0 & 1
\end{array}\right]
$$

\section{Error Model}

Assume that the model errors are very small and use $A_{i}$ to 
express the nominal transformation matrix from joint $i-1$ to joint $i ; A_{i}{ }^{\prime}$ then expresses the actual transformation matrix as:

$$
\begin{gathered}
A_{i}{ }^{\prime}=R_{X}\left(\alpha_{i-1}+\delta \alpha_{i-1}\right) \cdot D_{X}\left(a_{i-1}+\delta a_{i-1}\right) \\
\cdot R_{Z}\left(\theta_{i}+\delta \theta\right) \cdot D_{Z}\left(d_{i}+\delta d_{i}\right) \cdot R_{Y}\left(\beta_{i}+\delta \beta_{i}\right)
\end{gathered}
$$

The error of one joint is equal to the sum of errors caused by every model error[5]:

$$
\begin{gathered}
d A_{i}=\frac{\partial A_{i}}{\partial \alpha_{i-1}} \delta \alpha_{i-1}+\frac{\partial A_{i}}{\partial a_{i}} \delta a_{i-1}+\frac{\partial A_{i}}{\partial \theta_{i}} \delta \theta_{i} \\
+\frac{\partial A_{i}}{\partial d_{i}} \delta d_{i}+\frac{\partial A_{i}}{\partial \beta_{i}} \delta \beta_{i}
\end{gathered}
$$

Just let,

$$
\begin{aligned}
& Q_{a}=A^{-1} \frac{\partial A_{i}}{\partial \alpha_{i-1}}=\left[\begin{array}{cccc}
0 & 0 & 0 & c \theta c \beta \\
0 & 0 & 0 & -s \theta \\
0 & 0 & 0 & c \theta s \beta \\
0 & 0 & 0 & 0
\end{array}\right] \\
& Q_{\alpha}=\left[\begin{array}{cccc}
0 & -c \theta s \beta & -s \theta & -d s \theta c \beta \\
c \theta s \beta & 0 & -c \theta c \beta & -d c \theta \\
s \theta & c \theta c \beta & 0 & -d s \theta s \beta \\
0 & 0 & 0 & 0
\end{array}\right] \\
& Q_{d}=\left[\begin{array}{cccc}
0 & 0 & 0 & -s \beta \\
0 & 0 & 0 & 0 \\
0 & 0 & 0 & c \beta \\
0 & 0 & 0 & 0
\end{array}\right] \\
& Q_{\theta}=\left[\begin{array}{cccc}
0 & -c \beta & 0 & 0 \\
c \beta & 0 & s \beta & 0 \\
0 & -s \beta & 0 & 0 \\
0 & 0 & 0 & 0
\end{array}\right] \\
& Q_{\beta}=\left[\begin{array}{cccc}
0 & 0 & 1 & 0 \\
0 & 0 & 0 & 0 \\
-1 & 0 & 0 & 0 \\
0 & 0 & 0 & 0
\end{array}\right]
\end{aligned}
$$

Therefore the error of MDH model is:

$d A_{i}=A_{i}\left(Q_{a_{i-1}} \delta a_{i-1}+Q_{\alpha_{i-1}} \delta \alpha_{i-1}+Q_{d_{i}} \delta d_{i}+Q_{\theta_{i}} \delta \theta_{i}+Q_{\beta_{i}} \delta \beta_{i}\right)$

\subsection{SCARA Position Error Model}

For the SCARA robot, the final error model is:

$$
{ }_{0}^{4} A+d{ }_{0}^{4} A=\prod_{i=1}^{n}\left(A_{i}+A_{i} Q_{i} \delta_{i}\right)
$$

Since the geometric errors are very small, the higher-order terms can be neglected.

$$
d_{0}^{4} A=A_{1} Q_{1} \delta_{1} A_{2} A_{3} A_{4}+\cdots+A_{1} A_{2} A_{3} A_{4} Q_{4} \delta_{4}
$$

When the position errors or distance errors are considered, only the first three rows of the forth columns of $d_{0}^{4} A$ should be considered; in other words, the focus should be on the positions of the robot. Therefore, $B_{i}$ is defined as the coefficient matrix of the MDH model errors.

When robot error is measured, there may be errors in the measuring tools. If the tool coordinates parallel to the base frame are defined, they will always exist as $\delta x, \delta y, \delta z$ with respect to the measuring tools and never change. So the relationship of position errors with respect to the model error is:

$$
\begin{gathered}
{\left[\begin{array}{l}
d x \\
d y \\
d z
\end{array}\right]=\sum_{i=1}^{4} B_{i} \delta_{i}+B_{\text {tool }}\left[\begin{array}{c}
\delta x \\
\delta y \\
\delta z
\end{array}\right]} \\
\delta_{i}=\left[\begin{array}{lllll}
\delta a_{i-1} & \delta \alpha_{i-1} & \delta d_{i} & \delta \theta_{i} & \delta \beta_{i}
\end{array}\right]^{T}
\end{gathered}
$$

Where $\delta_{i}$ is the MDH model errors of the link $i-1$ to link $\mathrm{i}$ and $B_{i}$ is the coefficient matrix of $\delta_{i}$.

Equation(12) can be written as:

$$
\left[\begin{array}{lll}
d x & d y & d z
\end{array}\right]^{T}=B \delta
$$

\subsection{Distance error model}

Unlike the difficulties involved in building accurate base frame when using the position error model to calibrate the robot, the distance error model allows distance to be measured easily and accurately without the need for a pre-determined base frame. Accordingly, the distance error model is used here to calibrate the robot.

The measured distance when the robot is moving is $l_{R}$, the command distance of the robot is $l_{C}$, and $\Delta l=l_{R}-l_{C}$ is the distance error between the measured distance and the command distance. Therefore:

$$
\begin{aligned}
\left(\Delta l+l_{R}\right)^{2} & =\left(x_{R}(j)-x_{R}(i)+\Delta x(j)-\Delta x(i)\right)^{2} \\
& +\left(y_{R}(j)-y_{R}(i)+\Delta y(j)-\Delta y(i)\right)^{2} \\
& +\left(z_{R}(j)-z_{R}(i)+\Delta z(j)-\Delta z(i)\right)^{2}
\end{aligned}
$$

So the final distance error model can be written as:

$$
\begin{gathered}
\Delta l=\left(\frac{x_{R}(j)-x_{R}(i)}{l_{R}(i, j)}, \frac{y_{R}(j)-y_{R}(i)}{l_{R}(i, j)}, \frac{z_{R}(j)-z_{R}(i)}{l_{R}(i, j)}\right) \\
\times\left(B_{j}-B_{i}\right) \Delta q
\end{gathered}
$$

\section{Experiment and results}

\subsection{Experiment set-up}

The first step is to build the SCARA MDH model. The 
SCARA is a very simple robot with all of its links parallel and three of its four joints rotational. The process involved in building the MDH model is depicted in Figure 1 and Table 1 .

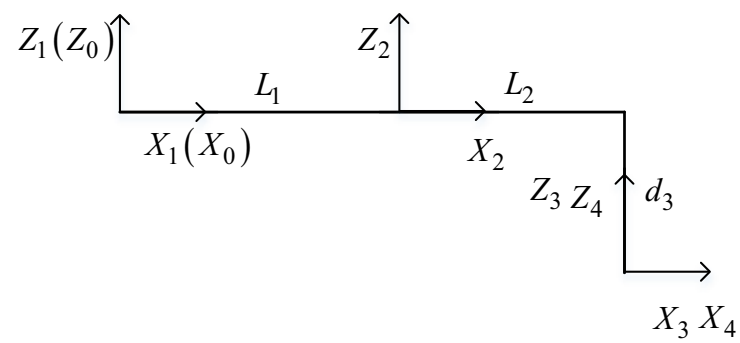

Figure 1. SCARA MDH model

Table 1.Nominal kinematic parameters

\begin{tabular}{|c|c|c|c|c|c|}
\hline Joint & $a_{i-1}$ & $\alpha_{i-1}$ & $d_{i}$ & $\theta_{i}$ & $\beta_{i}$ \\
\hline 1 & 0 & 0 & 0 & $\theta_{1}$ & 0 \\
\hline 2 & 180 & 0 & 0 & $\theta_{2}$ & 0 \\
\hline 3 & 180 & 0 & 0 & 0 & 0 \\
\hline 4 & 0 & 0 & 0 & $\theta_{4}$ & 0 \\
\hline
\end{tabular}

The end-effector could only be measured after the MDH model had been built. In this experiment, a FARO laser tracker with an accuracy of $10 \mathrm{um}+2.5 \mathrm{um} / \mathrm{m}$ was used for measuring the errors of the SCARA robot. In order to measure robot position error, a robot base frame was built to allow the robot to move in the XY plane and was fitted so that the $\mathrm{XY}$ plane normal vector is the $\mathrm{Z}$ axis. The base frame was completed by allowing the robot to move along the $\mathrm{X}$ axis so that the axis could be identified as such.

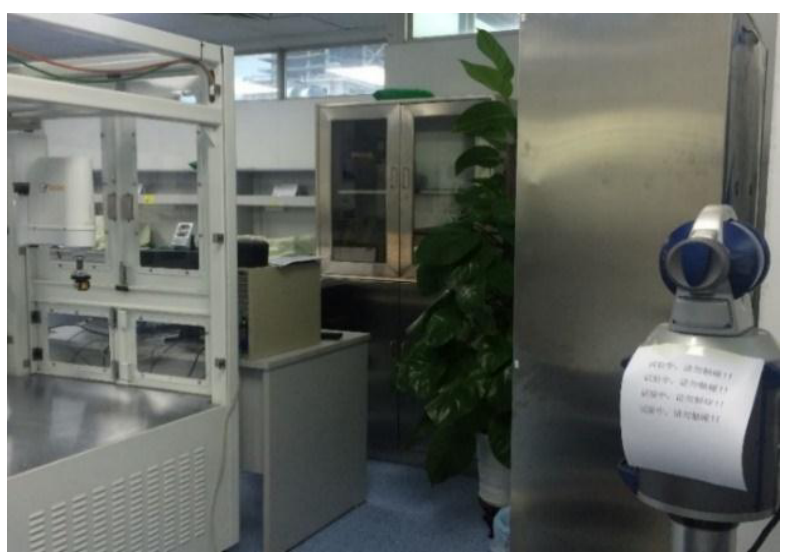

Figure 2.Experiment field

Two groups of positions of the robot end-effector were measured. The calibrated group was used to calibrate the robot, while the check group was to check the accuracy of the robot after calibration. A total of 88 positions were measured for each group.

Finally, the measured data are substituted into equation(13) and equation(15), resulting in a set of equations that are all linear. The least-square method can then be used to solve the equations.

\subsection{Position error model results}

The lease-square equation for the position error model was built according to equation (13) using the measured data:

$$
\boldsymbol{b}_{p}=C_{p} \Delta \boldsymbol{q}_{p}
$$

Where, $\boldsymbol{b}_{\boldsymbol{p}}$ is the position errors vector between the measured position and command position of the robot end-effector, $C_{p}$ is the coefficient matrix consisting of Jacobian matrix $B_{i}$, and $\Delta \boldsymbol{q}_{\boldsymbol{p}}$ is the MDH model errors.

Table 2.Calibration results of position error

\begin{tabular}{|c|c|c|c|c|c|}
\hline$i$ & $\delta a_{i-1}$ & $\delta \alpha_{i-1}$ & $\delta d_{i}$ & $\delta \theta_{i}$ & $\delta \beta_{i}$ \\
\hline 1 & 0 & 0.0002 & 0 & -0.0065 & 0.0027 \\
\hline 2 & -0.8340 & 0.0011 & 0 & 0.0105 & 0.0018 \\
\hline 3 & -0.2226 & 0.0003 & 0 & 0 & 0 \\
\hline tool & \multicolumn{4}{|c}{$\delta x=-0.8438 \delta y=-1.8113 \delta z=-18.5336$} \\
\hline
\end{tabular}

Table 2 is the MDH model errors after equation(16) had been calibrated. The absolute error of the end-effector before calibration and after calibration of the calibrated group pointsis shown in Figure 3.
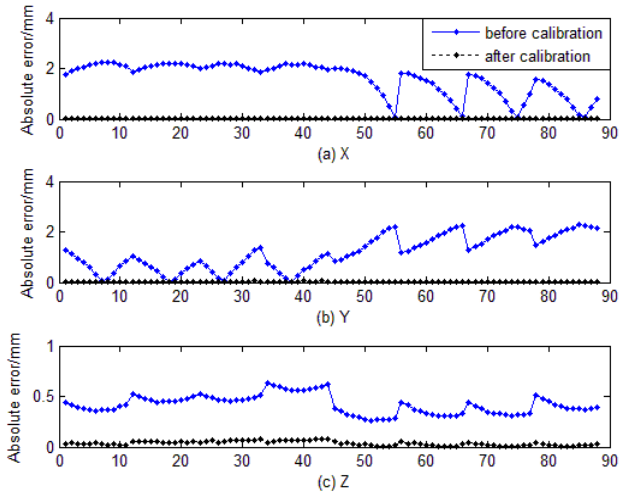

Figure 3.Position error of calibrated group

Because the model errors were calculated with the calibrated group points only, the improvement of accuracy may only be effective for the calibrated groups. To compensate for this another 88 points were measured as a check group in different positions. Their position errors after calibration are shown in Figure 4.

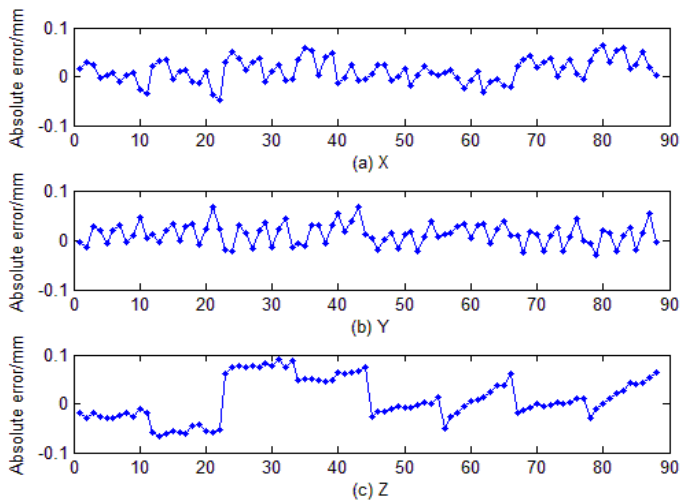

Figure 4.Position error of check group 
Table 3.Position error analysis

\begin{tabular}{|c|c|c|c|c|c|c|c|}
\hline \multirow{2}{*}{\multicolumn{2}{|c|}{ Error }} & \multicolumn{3}{|c|}{$\begin{array}{l}\text { Calibrated Group } \\
\text { Error }(\mathrm{mm})\end{array}$} & \multicolumn{3}{|c|}{$\begin{array}{l}\text { Check Group } \\
\text { Error }(\mathrm{mm})\end{array}$} \\
\hline & & $\mathrm{X}$ & $\mathrm{Y}$ & $\mathrm{Z}$ & $\mathrm{X}$ & $\mathrm{Y}$ & $Z$ \\
\hline \multirow{2}{*}{$\begin{array}{c}\text { BeforeCalibr } \\
\text { ation }\end{array}$} & $\begin{array}{l}\mathrm{Me} \\
\text { an }\end{array}$ & $\begin{array}{c}1.61 \\
01 \\
\end{array}$ & $\begin{array}{c}1.18 \\
01 \\
\end{array}$ & $\begin{array}{c}0.41 \\
73 \\
\end{array}$ & \multirow{2}{*}{\multicolumn{3}{|c|}{ NONE }} \\
\hline & SD & $\begin{array}{c}0.64 \\
38\end{array}$ & $\begin{array}{c}0.69 \\
31\end{array}$ & $\begin{array}{c}0.09 \\
43\end{array}$ & & & \\
\hline \multirow{2}{*}{$\begin{array}{c}\text { After } \\
\text { Calibration }\end{array}$} & $\begin{array}{l}\mathrm{Me} \\
\text { an }\end{array}$ & $\begin{array}{c}0.01 \\
73\end{array}$ & $\begin{array}{c}0.01 \\
81\end{array}$ & $\begin{array}{c}0.03 \\
61\end{array}$ & $\begin{array}{c}0.02 \\
14\end{array}$ & $\begin{array}{c}0.02 \\
04\end{array}$ & $\begin{array}{c}0.03 \\
59\end{array}$ \\
\hline & SD & $\begin{array}{c}0.01 \\
17\end{array}$ & $\begin{array}{c}0.01 \\
31\end{array}$ & $\begin{array}{c}0.02 \\
18\end{array}$ & $\begin{array}{c}0.01 \\
62\end{array}$ & $\begin{array}{c}0.01 \\
39\end{array}$ & $\begin{array}{c}0.02 \\
58\end{array}$ \\
\hline
\end{tabular}

Once the data was obtained, the results were analyzed and the position error expressed respectively in the $\mathrm{X}, \mathrm{Y}$ and $\mathrm{Z}$ axes. From Table 3 it can be seen that for the calibrated group points after the calibration the mean position errors could be decreased from $1.6101 \mathrm{~mm}$ to $0.0173 \mathrm{~mm}$ in the $\mathrm{X}$ axis; $1.1801 \mathrm{~mm}$ to $0.0181 \mathrm{~mm}$ in the $\mathrm{Y}$ axis; and $0.4173 \mathrm{~mm}$ to $0.0361 \mathrm{~mm}$ in the $\mathrm{Z}$ axis. The standard deviation (SD) could be improved from 0.6438 to 0.0117 in the $\mathrm{X}$ axis; 0.6931 to 0.0131 in the $\mathrm{Y}$ axis; and 0.0943 to 0.0218 in the $Z$ axis. For the check group points, the mean position errors are $0.0214 \mathrm{~mm}$ in the $X$ axis; $0.0204 \mathrm{~mm}$ in the $\mathrm{Y}$ axis; and $0.0359 \mathrm{~mm}$ in the $\mathrm{Z}$ axis. The SD in three axes respectively is $0.0162 \mathrm{~mm}$, $0.0139 \mathrm{~mm}$, and $0.0258 \mathrm{~mm}$. These data show that this method substantially improves robot positioning accuracy. However, the method requires that before measuring position errors it is necessary to build a robot base frame and confirm the relationship between the base frame and the measurement system. If the relationship is not accurate, it may cause other errors. To overcome this difficulty, a distance error model $[5,11]$ is proposed in the next section.

\subsection{Distance error model results}

When using the laser tracker as the measurement system, it is easy to confirm the base frame's accuracy because of the laser's high measurement accuracy. However, when it is not possible to get an accurate base frame, a distance error model can make it easier to calibrate the robot.

After the measurements are obtained, they can be put into equation(15), so that:

$$
\Delta \boldsymbol{l}_{\boldsymbol{d}}=D_{d} \Delta \boldsymbol{q}_{\boldsymbol{d}}
$$

Where, $\Delta \boldsymbol{l}_{\boldsymbol{d}}$ is the distance errors vector between the measured positions and command positions; $D_{d}$ is the coefficient matrix consisting of $B_{j}-B_{i}$, and $\Delta \boldsymbol{q}_{\boldsymbol{d}}$ is the MDH model errors.

After the robot is calibrated with the distance error, the results are as shown in Table 4.

Table 4.Results of distance error model

\begin{tabular}{|c|c|c|c|c|c|}
\hline$i$ & $\delta a_{i-1}$ & $\delta \alpha_{i-1}$ & $\delta d_{i}$ & $\delta \theta_{i}$ & $\delta \beta_{i}$ \\
\hline 1 & 0 & 0 & 0 & 0 & -0.0022 \\
\hline 2 & -0.9140 & -0.0015 & 0 & 0.0104 & -0.0042 \\
\hline 3 & -0.3299 & 0.0014 & 0 & 0 & 0 \\
\hline
\end{tabular}

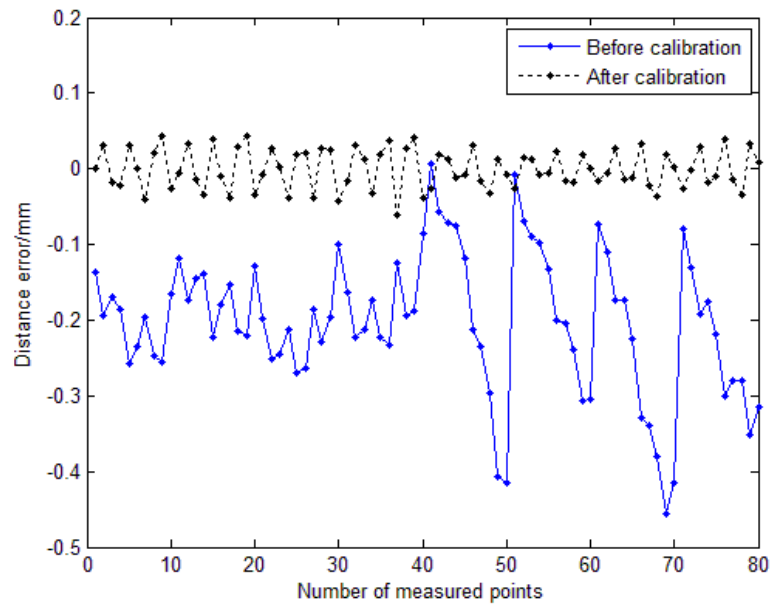

Figure 5.Distance error of calibrated group

The robot can be corrected for distance errors with the calibration parameters: the distance errors before calibration and after calibration can be seen in Fig. 5. Just as with the position error, the calibration may only improve the accuracy of the calibrated group, so it is necessary to measure distance errors of other points that are different to the calibrated group points. Therefore, 88 points, which is 80 distance errors, were measured and are shown in Fig. 6.

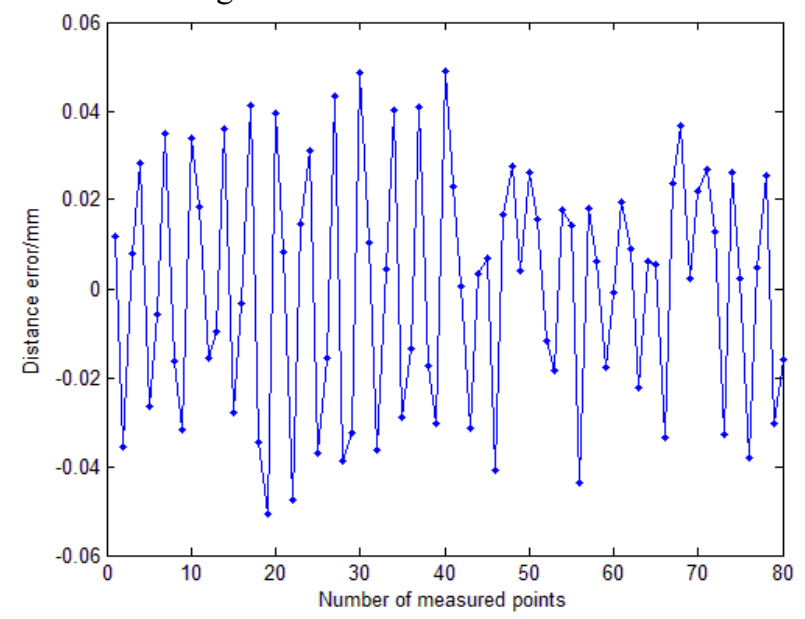

Figure 6.Distance error of check group

Table 5.Distance error analysis

\begin{tabular}{|c|c|c|c|}
\hline \multicolumn{2}{|c|}{ Error } & $\begin{array}{c}\text { Calibrated } \\
\text { Group }\end{array}$ & $\begin{array}{c}\text { Check } \\
\text { Group }\end{array}$ \\
\hline \multirow{2}{*}{ BeforeCalibration } & Mean & 0.2031 & \multirow{2}{*}{ None } \\
\cline { 2 - 4 } & SD & 0.0928 & 0.0230 \\
\hline \multirow{2}{*}{ After Calibration } & Mean & 0.0225 & 0.0268 \\
\cline { 2 - 4 } & SD & 0.0260 & 0.006 \\
\hline
\end{tabular}

From Figure 6 and Table 5 it can be seen that for the calibrated group points after the calibration, the mean distance errors decreased from $0.2031 \mathrm{~mm}$ to $0.0225 \mathrm{~mm}$, the standard deviation (SD) improved from $0.0928 \mathrm{~mm}$ to $0.0260 \mathrm{~mm}$, and the check group points' mean distance error and SD are $0.0230 \mathrm{~mm}$ and $0.0268 \mathrm{~mm}$ respectively.

From these data it is clear that the distance error model works very well and can be used as a method when it is not 
possible to construct an accurate base frame.

\section{Conclusion}

This paper described the building of a SCARA robot model based on the MDH model, and proposed two error models for calibrating the robot. After the robot was calibrated, the parameters were used to correct the robot, which greatly improved the robot's accuracy. When it is not possible to build an accurate robot base frame and establish the relationship of the base to the measurement system, it is suggested to use the distance error model to achieve accuracy. Both error models proved to be effective for calibrating the SCARA robot based on an accurate robot base frame.

\section{Acknowledge}

The authors gratefully acknowledge the support from the National Nature Science Foundation of China under the NSFC Grant 51375118, and the support from Shenzhen Government Founding GJHZ20140419142750948.

\section{References}

1 M. Švaco, B. Šekoranja, F. Šuligoj, et al., Calibration of an Industrial Robot Using a Stereo Vision System, Procedia Engineering, 69, 459-463 (2014)

2 S. A. Hayati, Robot arm geometric link parameter estimation, Decision and Control, 1983. The 22nd IEEE Conference on, 1477-1483 (1983)

3 A. Nubiola, I. A. Bonev, Absolute calibration of an ABB IRB 1600 robot using a laser tracker, Robotics and Computer-Integrated Manufacturing, 29, 236-245 (2013)
4 A. Nubiola, I. A. Bonev, Absolute robot calibration with a single telescoping ballbar, Precision Engineering, 38, 472-480 (2014)

5 W. Zhenhua, X. Hui, C. Guodong, et al., A distance error based industrial robot kinematic calibration method, Industrial Robot: An International Journal, 41, 439-446 (2014)

6 J. Zhou, H. N. Nguyen, H. J. Kang, Selecting Optimal Measurement Poses for Kinematic Calibration of Industrial Robots, Advances in Mechanical Engineering, 6, 291389-291389 (2015)

7 J. Santolaria, J.-A. Yagüe, R. Jiménez, et al., Calibration-based thermal error model for articulated arm coordinate measuring machines, Precision Engineering, 33, 476-485 (2009)

8 A. Nubiola, M. Slamani, A. Joubair, et al., Comparison of two calibration methods for a small industrial robot based on an optical CMM and a laser tracker, Robotica, 32, 447-466 (2013)

9 A. Joubair, M. Slamani, I. A. Bonev, Kinematic calibration of a five-bar planar parallel robot using all working modes, Robotics and Computer-Integrated Manufacturing, 29, 15-25 (2013)

10 G. Du, P. Zhang, Online robot calibration based on vision measurement, Robotics and Computer-Integrated Manufacturing, 29, 484-492 (2013)

11 A. Joubair, I. A. Bonev, Kinematic calibration of a six-axis serial robot using distance and sphere constraints, The International Journal of Advanced Manufacturing Technology, 77, 515-523 (2014)

12 N. Xi, Y. Liu, Low-cost and automated calibration method for joint offset of industrial robot using single-point constraint, Industrial Robot, 38, 577-584 (2011) 